\title{
Donner une seconde vie aux déchets électroniques
}

Économies informelles et innovation sociotechnique par les marges

Re-making Electronic Waste: Informal Economies and Socio-Technical Innovation from the Margins

\section{Blanca Callén}

\section{OpenEdition Journals}

Édition électronique

URL : https://journals.openedition.org/tc/7962

DOI : $10.4000 /$ tc. 7962

ISSN : 1952-420X

Éditeur

Éditions de l'EHESS

\section{Édition imprimée}

Date de publication : 31 octobre 2016

Pagination : 206-219

ISBN : 9782713225291

ISSN : 0248-6016

\section{Référence électronique}

Blanca Callén, «Donner une seconde vie aux déchets électroniques », Techniques \& Culture [En ligne], 65-66 | 2016, mis en ligne le 31 octobre 2018, consulté le 29 septembre 2022. URL : http:// journals.openedition.org/tc/7962; DOI : https://doi.org/10.4000/tc.7962 


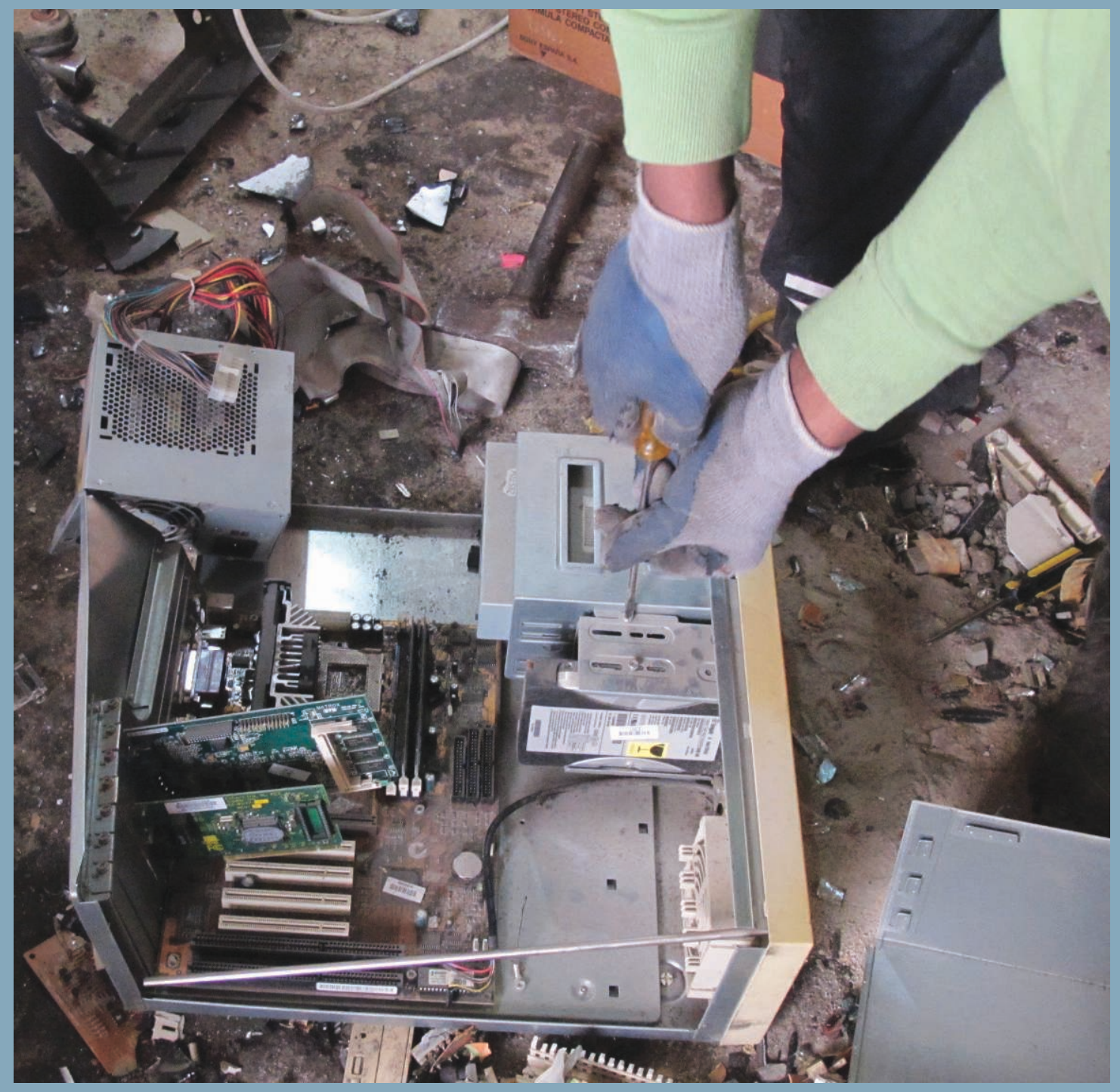




\section{Donner une seconde vie aux déchets électroniques} Économies informelles et innovation
sociotechnique par les marges

Au sein des imaginaires collectifs d'un présent et d'un futur fantasmés, le lien entre progrès, innovation et technologie est clairement établi. Les appareils technologiques et les gadgets dernier cri sont envisagés comme des solutions prometteuses à nos besoins quotidiens, aux problèmes sociaux et aux enjeux publics. Dans le même temps, le marché mondial de l'équipement électrique et électronique «grandit de manière exponentielle, tandis que la durée de vie des produits est toujours plus courte» (Khurrum et al. 2011). Par conséquent, nous sommes confrontés aux conséquences néfastes de cette omniprésence de la technologie: les déchets électroniques (e-waste).

Afin de remédier à ce problème, l'Union européenne (UE) a émis une directive ${ }^{1}$ visant à empêcher l'accumulation des déchets électroniques et électriques, et à promouvoir la récupération, le recyclage et la réparation de ces objets. Cette politique s'appuie sur le principe de la responsabilité élargie du producteur (REP), qui encourage les efforts en matière d'environnement via la responsabilité accrue des fabricants tout au long de la vie du produit, et tout particulièrement au moment de sa mise au rebut, de son recyclage et de son élimination définitive. Ce projet politique présente toutefois un certain nombre de lacunes. En observant plus attentivement la phase de recyclage, on note un manque de culture écologique, alors qu'une telle mesure devrait seulement être la dernière étape du cycle. Elle constitue par ailleurs un bon exemple de politique «descendante» fondée sur une logique technocratique : le rôle des techniciens, de l'industrie et des autorités gouvernementales est au centre, tandis que les citoyens sont vus comme de simples consommateurs, responsables de leurs propres déchets. En pratique, le principe du REP oblige les producteurs à prendre en charge le processus de recyclage et soumet tous les appareils achetés à une taxe inexpliquée et invisible. En théorie, cette taxe est destinée à financer l'ensemble du processus de collecte et de valorisation de déchets, de leur évaluation, de leur transfert à une usine de traitement et de recyclage dans de bonnes conditions écologiques et sanitaires. En réalité, ce n'est pas toujours le cas. 
Le premier obstacle est l'invisibilité de nombreux producteurs: «Sur les 10000 producteurs [d'appareils électroniques] supposés exister en Espagne, seuls 1000 , environ, sont enregistrés » (Queiruga et al. 2012: 62). Par conséquent, la gestion des déchets d'équipements électriques et électroniques (DEEE) produits par la population espagnole doit être soutenue par seulement $10 \%$ des producteurs, et il est impossible de contrôler convenablement le traitement des déchets électroniques dans son ensemble. En 2011, des recherches ayant prouvé que tous les appareils placés dans des points de collecte municipaux n'étaient pas correctement recyclés, et que nombre d'entre eux échappaient aux circuits officiels, l'Organisation espagnole des consommateurs et des usagers (OCU) a dénoncé la situation, parlant même de fraude: alors que les consommateurs déboursent 300 millions d'euros par an pour la gestion des DEEE, seuls $10 \%$ sont correctement perçus et réellement financés par les producteurs. Où passe alors la différence entre les fonds levés et les frais finaux payés? ${ }^{2}$

L'existence prouvée d'évasions et de fuites illégales dans le circuit institutionnel de la gestion et du traitement des DEEE est à l'origine de ma problématique de recherche: que se passe-t-il en dehors du circuit officiel? Quels rôles jouent les usagers, les consommateurs et les groupes informels? Quelles solutions alternatives créatives et innovantes visant à réduire les déchets électroniques apparaissent? D'une certaine façon, si l'on envisage les usagers, les consommateurs et les groupes informels comme étant des experts potentiels sur ces problèmes qui les touchent directement, quelles sont les stratégies officieuses employées une fois qu'un ordinateur est considéré comme un déchet? Pour répondre à ces questions, je me concentrerai sur l'une des trois ${ }^{3}$ expériences qui ont constitué mon travail ethnographique de terrain, consistant à intégrer un groupe de ramasseurs de déchets et de ferrailleurs - la plupart étant des immigrés illégaux en provenance d'Afrique subsaharienne- qui vivent et travaillent à Barcelone ${ }^{4}$.

\section{Récupération}

Le travail des ramasseurs de déchets commence avec la collecte des restes provenant des décharges publiques des environs. Bien qu'il existe des centres de recyclage un peu partout dans la ville destinés aux ordures ménagères recyclables (verre, papier/carton, plastique, déchets organiques, etc.), et bien que pour chaque quartier soit fixé un horaire hebdomadaire durant lequel les gens peuvent laisser leurs meubles et appareils dans la rue pour être collectés par les services municipaux, nombreux sont ceux qui n'y font pas appel. Il est extrêmement fréquent que les gens laissent leurs objets inutiles sur la voie publique en dehors des heures de collecte, afin que d'autres puissent les ramasser et les réutiliser. En conséquence de quoi, un réseau informel et discret d'échange et de réutilisation des déchets est apparu. Tout au long de la journée et à travers toute la ville - mais en particulier dans les quartiers où un service de collecte des déchets s’apprête à être lancé-, il est fréquent de voir des ramasseurs informels déambulant dans les rues. Leurs trouvailles - en particulier en métal ${ }^{5}$ - proviennent du hasard, de la générosité des uns ou 
par arrangement pour les autres, et d'une connaissance approfondie développée au fil des jours, à force d'observation et de confrontation aux marges de la société.

«Je n’ai jamais accepté le mot "ordure", déclare un récupérateur de déchets, car tout ce qui est ramassé dans la rue a de la valeur. » Aux capacités sensorielles permettant de déceler les métaux parmi les ordures s'ajoute une aptitude à calculer rapidement et à redonner à l'objet une certaine valeur économique. L'intérêt de la récupération d'objets, par conséquent, réside non seulement dans leur simple reconnaissance mais aussi dans leur transformation virtuelle, en ayant recours à l'imagination pour dépasser l'aspect actuel de l'objet et de visualiser toutes ses potentialités, y compris sur le plan économique, car «ce qui est la fin pour quelqu'un peut être le début pour quelqu'un d'autre» (Lepawsky \& Mather 2011 : 246).

Cette activité de récupération requiert en outre une capacité à composer avec la municipalité: il s'agit non seulement de se tenir informé des horaires de collecte des déchets dans chaque quartier -le cas de figure idéal - mais aussi de défier les autorités, dans la mesure où l'utilisation des espaces urbains est régie par une ordonnance municipale ${ }^{6}$, laquelle qualifie «le ramassage et le retrait de déchets déposés sur la voie publique » d'infraction mineure, passible d'une amende pouvant aller jusqu'à 450,76 euros (BOPB 2001). Toutefois, un certain nombre de ramasseurs de déchets ont expliqué que cela arrive rarement, la police pouvant tantôt se montrer menaçante, tantôt fermer les yeux. Si cela n'était pas le cas, une crise socio-économique majeure éclaterait chez les migrants se retrouvant sans moyens pour survivre dans la jungle urbaine. En d'autres termes, le ramassage de déchets pourrait être vu comme une activité illégale mais efficace et durable pour gérer les conflits sociaux et les problèmes urbains et sanitaires auxquels les autorités locales sont confrontées.

\section{Commerce de la ferraille}

Les ramasseurs de déchets, qui squattaient et avaient établi leur commerce dans un grand complexe de magasins d'une zone défavorisée, vendaient leur récolte quotidienne de métal aux ferrailleurs du quartier -les intermédiaires-, qui vivaient et travaillaient dans certains des plus grands entrepôts. Il s'agissait de ramasseurs de déchets qui s'étaient installés là depuis plus longtemps et avaient pris les meilleures places, près de l'entrée. Manifestement, cet endroit offrait plus d'espace pour acheter, stocker et vendre la ferraille et le métal à un prix plus élevé ${ }^{7}$ aux commerçants légaux, qui à leur tour revendaient les pièces à des grosses fonderies. Les entrepôts les plus éloignés de l'entrée - un culde-sac-jouissaient de moins belles opportunités commerciales, et servaient simplement de lieux de travail ou de lieux de vie, remplis de baraques de fortune construites par les habitants eux-mêmes.

Pour chaque objet ramassé, et en particulier pour les objets, appareils électriques ou électroniques intacts, les ramasseurs de déchets effectuaient un calcul rapide et précis de la façon dont ils pouvaient en tirer le plus de valeur (monétaire) possible. Il fallait qu'ils sachent à la fois comment et à quel endroit ouvrir l'objet, repérer l'emplacement des composants et de 
1. Trousse à outils d'un ramasseur de déchets informel: gants, outils, un aimant (cercle en métal), des fruits, une paire de ciseaux et un livre d'exégèse biblique.

2. Tableau noir affichant les prix que les ferrailleurs du quartier payent pour chaque type de matériau: fer, aluminium, piles/batteries, acier et alliages de métaux.
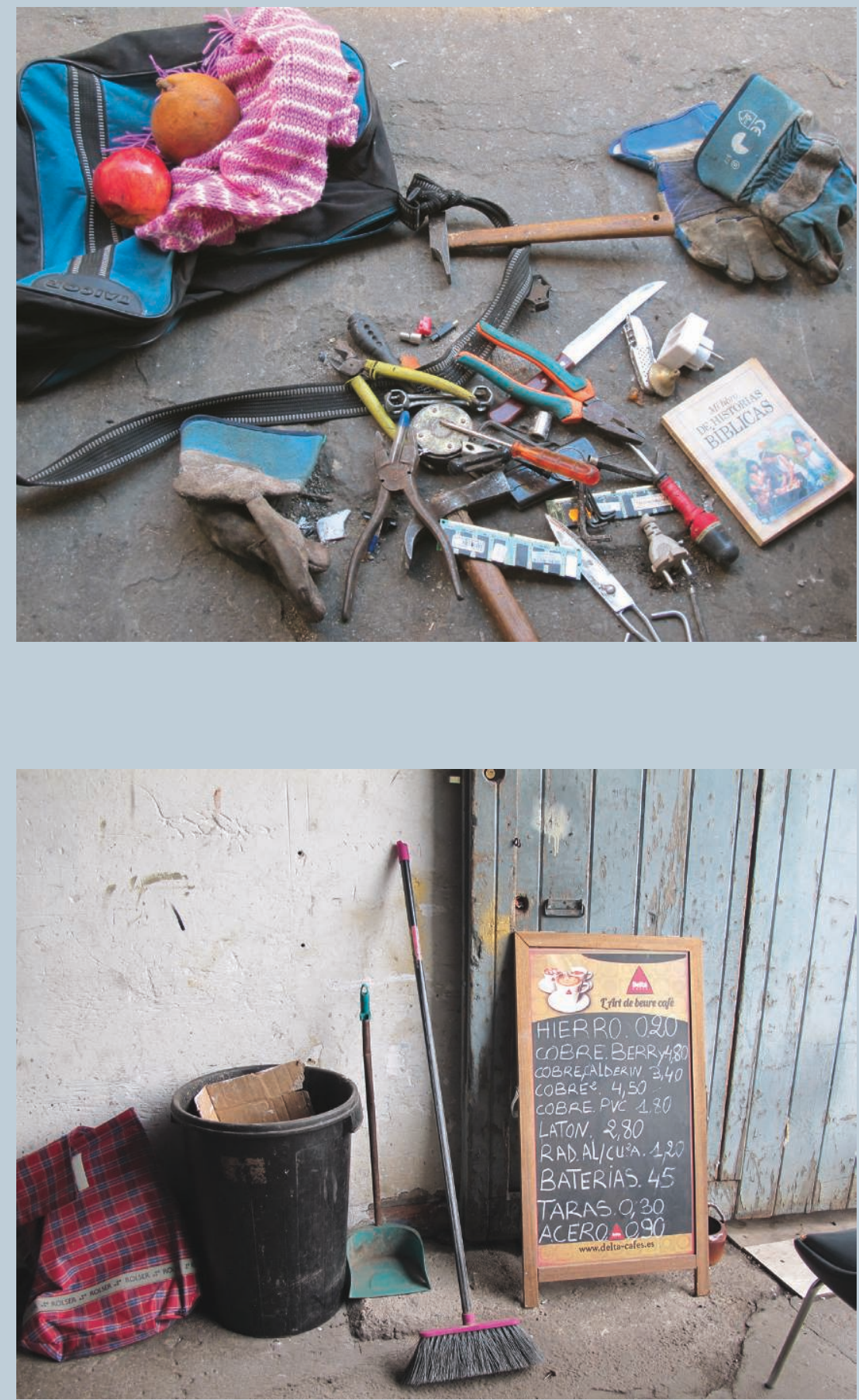


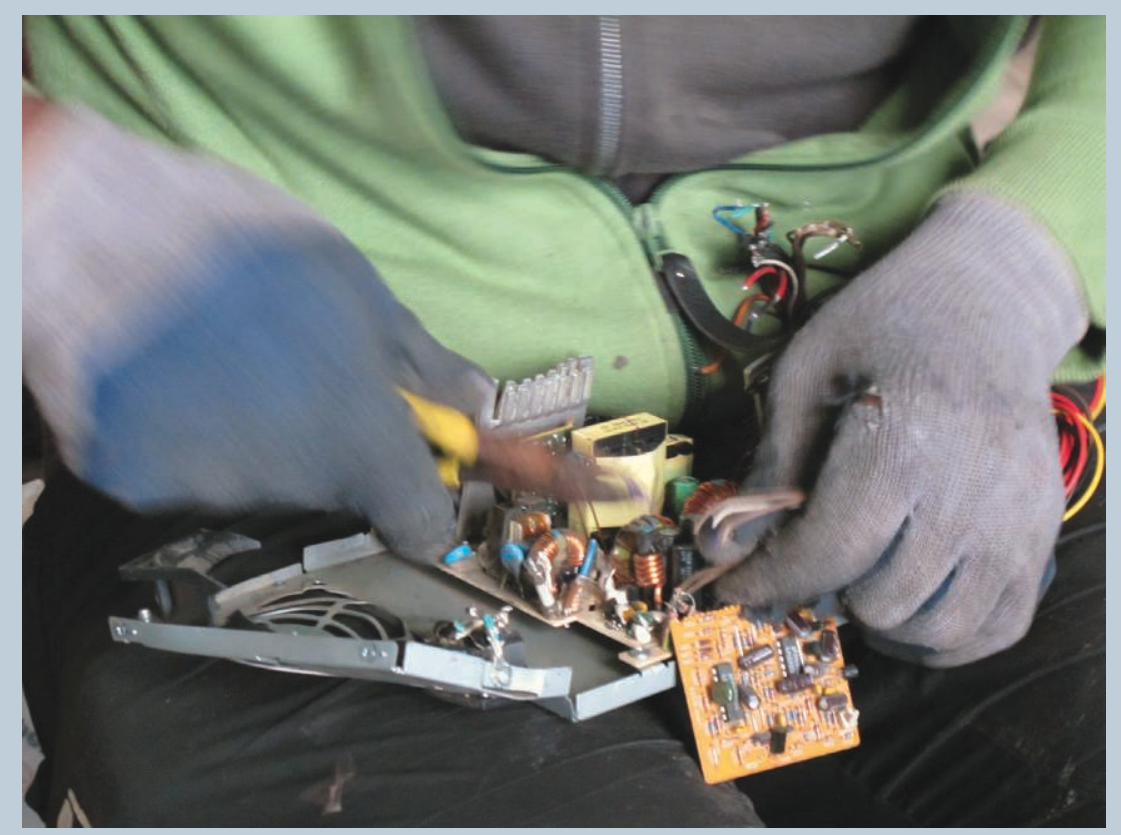

Extraction de pièces de cuivre d'une carte mère.

4. Test de branchement pour vérifier si un micro-ondes fonctionne.

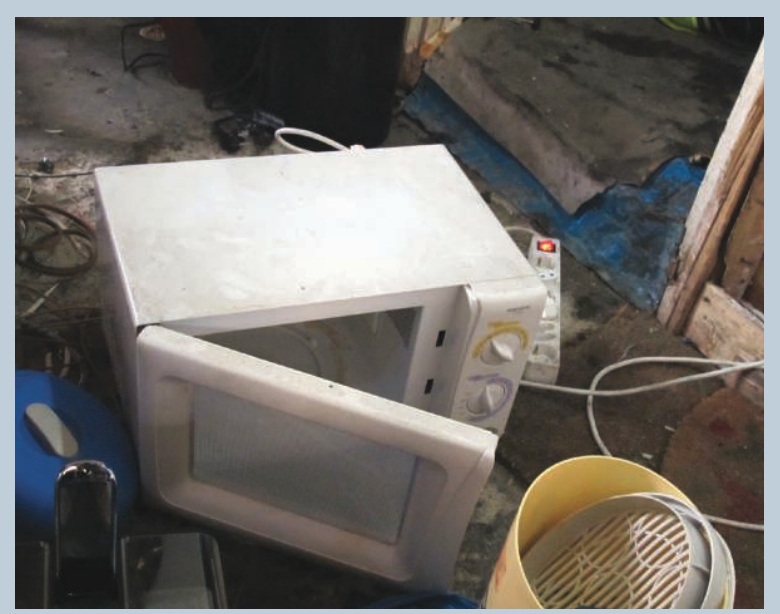

5esée de la collecte de la journée sur une balance électrique à l'intérieur de l'entrepôt appartenant à un intermédiaire du quartier

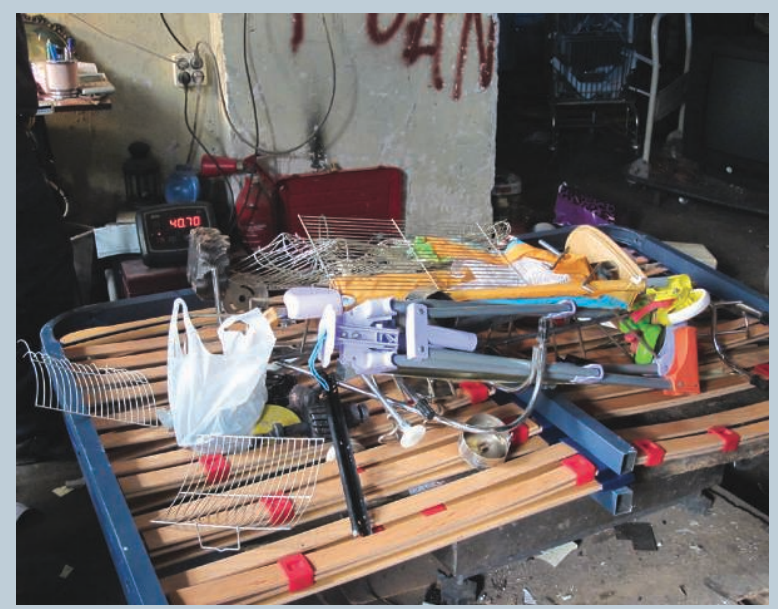


quels matériaux ils étaient constitués. Ces diverses manipulations étaient menées une fois de retour à l'entrepôt. Notre informateur, Marcel, consacrait tous ses après-midi et ses soirées au ramassage, car il s'agissait des moments de la journée où les gens étaient les plus nombreux à se débarrasser de leurs déchets, tandis qu'il passait ses matinées à démonter les objets ramassés la veille. Il était important d'agir vite et proprement, car une ouverture mal calculée ou un coup donné au mauvais endroit pouvaient rendre l'opération bien plus difficile. «On ne sait jamais ce qu'on peut trouver à l'intérieur», m'avait dit Marcel en examinant une vieille lampe. L'objet le plus indispensable pour les ramasseurs de déchets était un aimant: si un morceau de métal « réagissait », c'est qu'il contenait du fer. En cas de non-réaction, le gratter permettait de distinguer le laiton couleur jaunâtre de l'aluminium. Le matériau possédant la plus grande valeur est le cuivre, caractérisé par sa couleur rougeâtre. Le poids d'un objet est aussi une caractéristique essentielle pour estimer s'il contient ou non un moteur ou des bobines de cuivre.

Dans le cas des appareils électroniques, brancher l'engin était un moyen de savoir s'il fallait le garder intact ou le cannibaliser ${ }^{8}$. S'il n'émettait aucun bruit étrange, et/ou si le bouton d'allumage s'éclairait, l'appareil pouvait être vendu entier. Pour les ordinateurs fonctionnant mal, on procédait directement à son démembrement, en établissant clairement une hiérarchie de valeurs: le transformateur électrique était la pièce la plus précieuse, car il s’agit de celle qui contient le plus de cuivre; venait ensuite le disque dur, tant pour l'aluminium qu'il contient que pour le revendre; enfin, les petites pièces en cuivre soudées à la carte mère. Les matériaux ferreux et de rebut étaient vendus quotidiennement aux ferrailleurs des environs, afin de gagner l'argent nécessaire chaque jour pour survivre. Tous les autres matériaux étaient stockés jusqu'à ce que le volume cumulé soit suffisant pour atteindre un prix plus élevé, avec le risque cependant que les stocks soient volés.

\section{Appareils en état de marche vendus sur les marchés d'occasion}

Les ordinateurs et les appareils électriques en apparent état de marche étaient envoyés vers des marchés d'occasion dans les pays d'Afrique dont étaient originaires les ramasseurs de déchets. Ils étaient réparés puis revendus, car, d'après Keita, un intermédiaire originaire du Sénégal, « c'est à ce moment qu'ils acquièrent de la valeur ». «En Afrique, on répare tout, mais pas en Europe: ici, on se contente de changer les pièces, car ça rapporte plus d'argent et permet d'économiser», expliquait un ramasseur de déchets. «Le capitalisme, c'est ça: l'objet est cassé, tu le jettes et tu en rachètes un autre», proclamait un autre. En raison des inégalités économiques mondiales et des différents régimes de valeurs, on assiste à différents endroits à une renaissance des circuits de recyclage des objets en fin de vie. C'est le cas notamment des téléviseurs à tube cathodique (CRT), qui ont été massivement supplantés en Europe par les écrans plats, mais sont encore en vente sur certains marchés africains.

Dans le complexe d'entrepôts étudié, ces appareils étaient vendus à un groupe informel de vendeurs marocains ou à Keita, qui était devenu un petit ferrailleur travaillant entre Barcelone et le Sénégal. Pour les Marocains, la facilité d'accès à leurs entrepôts, le soutien mutuel et le caractère 
collectif de leur entreprise, le nombre de partenaires ainsi que la proximité géographique avec le Maroc facilitaient leur activité d'exportation de seconde main. Malgré tous les obstacles, de nombreux autres ramasseurs de déchets rêvaient également de devenir des négociants internationaux. Lopération nécessitait de mettre suffisamment d'argent de côté pour acheter un vieux fourgon et le remplir de vêtements et d'appareils soigneusement sélectionnés, en direction de leurs pays natals, payer des droits de douane et construire un réseau de gens de confiance pour veiller à ce que la marchandise arrive à bon port, qu'elle soit distribuée sur les marchés d'occasion et payée. Pour Keita, la destination était le Sénégal, et pour notre principal informateur, Marcel, c'était le Cameroun.

Au cours de notre travail de terrain, Keita avait obtenu une fourgonnette et était sur le point d'en acheter une seconde. En parallèle, il stockait et classait, à l'intérieur de l'entrepôt, des antiquités, des vêtements et chaussures, des appareils et ordinateurs de toutes sortes. Sa situation économique et son statut juridique, plus favorable que d’autres (il était autorisé à vivre et travailler en Espagne), lui facilitaient la tâche pour devenir commerçant. Labsence d'autorisations officielles n'avait pas empêché Marcel ${ }^{9}$ et d'autres d'entreposer les appareils dans l'intention de travailler eux aussi dans l'export.

Les marchandises sélectionnées pour le futur voyage vers l'Afrique devaient répondre à certaines exigences telles que la demande des marchés, les modes locales, la concurrence internationale et la possibilité d'obtenir un fourgon. Àtitre d'exemple, plus personne ne voulait de claviers d'ordinateurs ou de souris et les clients marocains étaient en demande de téléviseurs argentés, mais pas noirs. Certaines marques gagnaient plus d'argent que d'autres, se positionnant généralement au même rang que les marques européennes. Le besoin en chaussures pour femmes sur les marchés sénégalais et camerounais était déjà comblé par les sociétés chinoises, et les frigos devaient être démontés, les moteurs partant à l'étranger tandis que le reste était vendu comme ferraille à Barcelone.

\section{Réparation et restauration}

Tilan était un ramasseur de déchets éthiopien de 28 ans qui, bien que vivant en Europe depuis plus de treize années, n’avait toujours pas de permis de travail ni de séjour. Il avait appris quelques rudiments d'informatique simplement en regardant et en posant des questions à un groupe de Tchèques calés en informatique qui remettaient en état des ordinateurs, et vivaient avec lui dans un précédent squat à Barcelone. Cette compétence rare le rendant très utile, il était devenu une sorte de vendeur et réparateur « officiel» des ordinateurs d'autres ramasseurs de déchets. De temps en temps, il travaillait au noir pour une boutique informatique, leur fournissant des pièces de rechange trouvées dans la rue et faisant pour eux quelques réparations: «Ils vont me payer trois fois moins que ce qu'ils payent par internet », m’avait-il dit, faisant référence à la somme qu'il avait obtenue des composants.

Il acquérait des processeurs via d'autres ramasseurs de déchets pour environ 5 euros et, après remise en état, il pouvait les revendre 30,40, voire 50 euros. Il installait également des programmes et faisait de la maintenance informatique en freelance pour d'autres ramasseurs de déchets et collègues, pour le prix de 10 ou 15 euros. «Si tu arrives à les réparer, alors ça vaut la peine. 


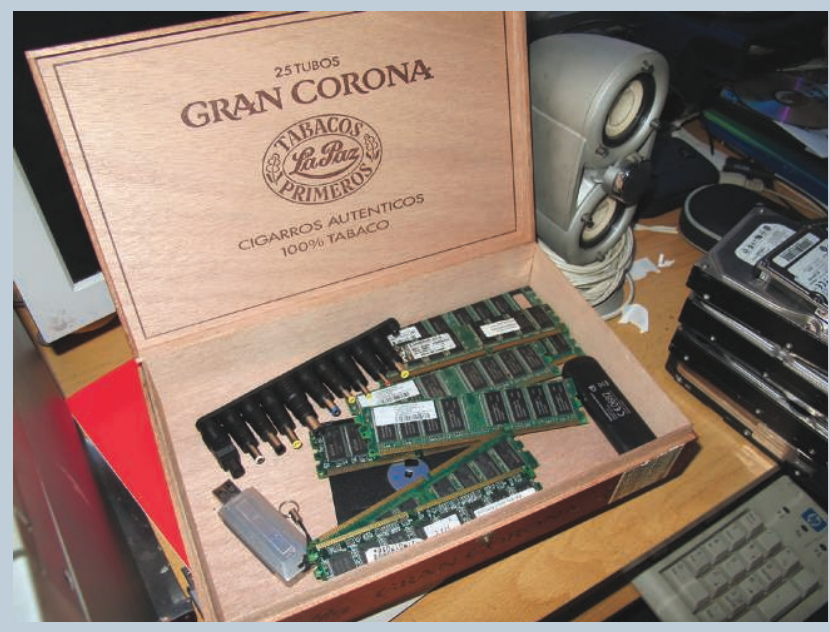

6. Boîte de Tilan contenant des mémoires centrales de rechange, destinées à être réutilisées dans le cadre d'une réparation ou d'une remise en état d'ordinateurs.

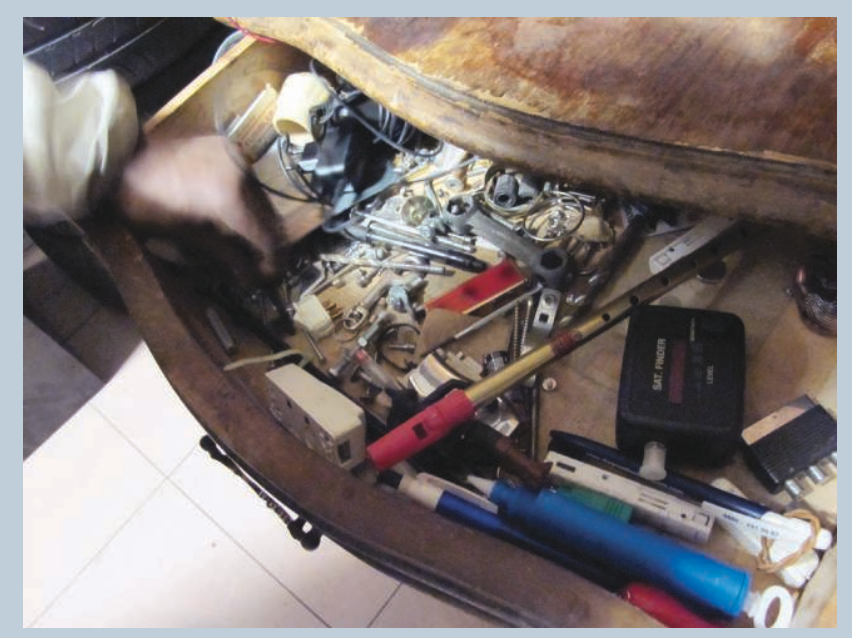

7. Tiroir à outils de Tilan.

8. Tilan en train de réparer une carte CPU pour la

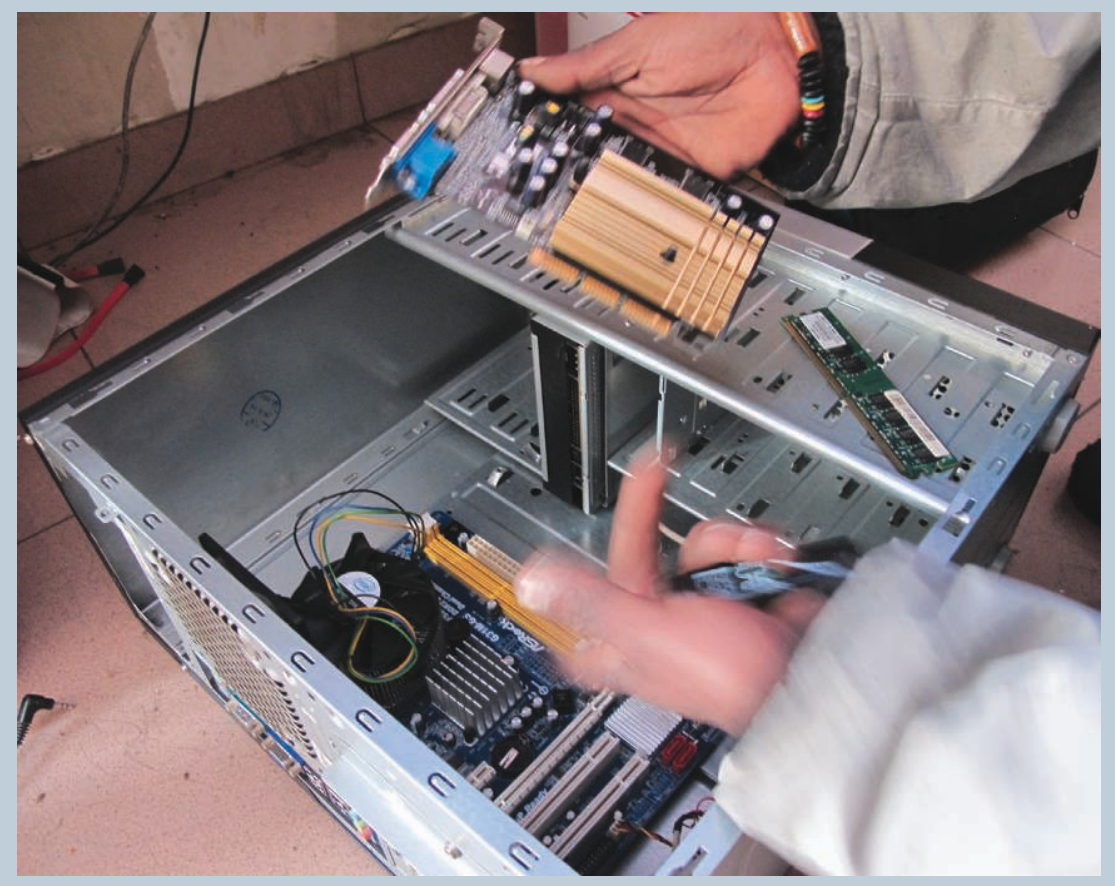


Mais si je laisse passer l'occasion, ils vont le détruire (le transformer en déchet) pour seulement 3euros », s'était-il plaint à propos des autres ramasseurs de déchets dépourvus de ses compétences en matière de réparation. Il donnait également une motivation écologique à son travail: «20 ordinateurs de sauvés, ce sont 20 ordinateurs en moins qui doivent être fondus ou brûlés. » Contrairement à ses collègues qui évaluaient les objets ramassés au poids, Tilan évaluait les ordinateurs et les appareils électroniques en fonction de la taille de leur mémoire ou de leur fonctionnalité. Pour reprendre les termes d'Ingold (2013), il était plus désireux de « conserver la forme» que de «donner forme».

Toutes les réparations et les restaurations de Tilan commençaient par un examen à l'aide d'une méthode par essais et corrections successifs, basée sur des pratiques sensorielles (Dant \& Bowles 2003, Dant 2010). Guidé par les «symptômes» tels que les différents bruits et autres réponses perceptibles, Tilan isolait les différentes variables jusqu'à ce qu'il parvienne à un diagnostic. «Je regarde si ça s'allume ou pas, si la tour fonctionne mais pas l'écran. [...] Si des lettres apparaissent sur l'écran, ça veut dire que c'est réparé à $50 \%$, et même plus si on peut entrer dans le BIOS. » «Grâce au son que ça fait, on peut souvent identifier le problème. Alors il suffit de retirer ce qui ne marche pas et de le remplacer par des pièces en bon état. Et si ça ne siffle pas, alors ça marche [... Mais] quand la carte mère est morte, tout est fichu et ça devient impossible à réparer », expliquet-il au sujet de ce système de réparation non « conventionnel» (Denis \& Pontille 2014).

Comparé au test que Marcel effectuait, consistant simplement à brancher les appareils qu'il avait ramassés, il s'agissait là d'un niveau de maîtrise bien plus poussé: les connaissances et compétences en informatique de Tilan lui permettaient de diagnostiquer des défaillances bien plus précises. Cette répartition inégale des connaissances épistémiques se traduisait à la fois par des écarts de revenus et par une division hiérarchique des statuts professionnels entre les habitants de l'entrepôt. Tandis que Tilan passait la plupart de son temps à acheter, réparer et vendre les pièces et les appareils électroniques intacts, ramassant les déchets uniquement lorsqu'il avait rapidement besoin d'argent, la plupart de ses collègues devaient consacrer davantage de temps à déambuler dans les rues de la ville pour ramasser, démonter et vendre leurs objets aux ferrailleurs officieux. Faisant partie d'un système pyramidal, seuls les intermédiaires comme Keita ou le groupe de Marocains pouvaient vendre leurs matériaux directement aux entreprises légales de récupération de ferraille au sommet de cette économie.

À travers ces trajectoires informelles, les déchets métalliques et la ferraille qui, à l'origine, échappaient au processus de traitement et de recyclage institutionnel ont désormais intégré le circuit légal du commerce de ferraille, pour arriver finalement dans les fonderies. Les appareils électroniques en état de marche ou potentiellement utilisables mis en décharge dans la banlieue de Barcelone ont emprunté une voie similaire: leur statut est passé de déchets dangereux et inutilisables à des ressources technologiques utiles, après réparation, au sein du circuit économique ravivé sur les marchés d'occasion africains. Tout objet entreposé dans l'attente de ce voyage tant espéré pouvait entre-temps être vendu directement sur place, à l'entrepôt. Toutes les occasions de vendre représentaient une bonne opportunité de gagner de l'argent, et ne devaient pas être manquées au profit de futures occasions plus risquées. 


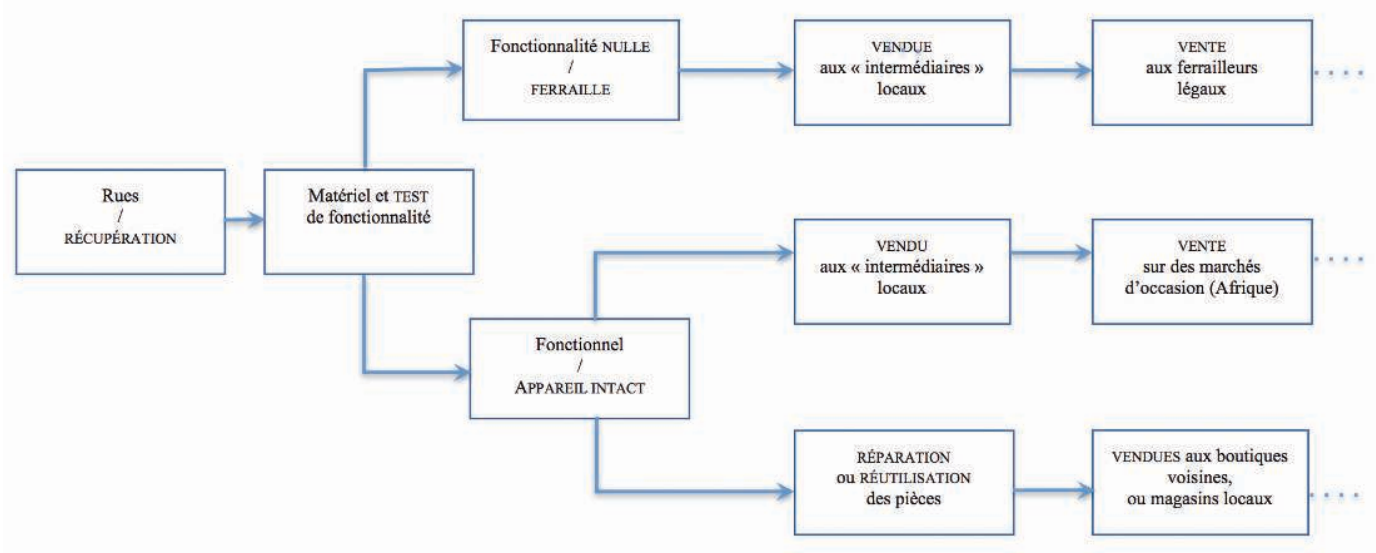

En redonnant vie aux déchets électroniques par une série de pratiques commençant par la récupération des objets, ces ramasseurs défient la destinée et l'ontologie des déchets électroniques, en leur (re)donnant de la valeur en fonction de leur nature et de leur état. Ils démontrent ainsi que les propriétés, les fonctions et les matériaux de ces objets ne sont pas des attributs fixes ou inhérents, mais ont au contraire une identité processuelle et relationnelle, et sont dotés d'un riche potentiel. Entre leurs mains, matériaux comme objets n'existent pas une fois pour toutes en tant qu'entités ou propriétés, mais « apparaissent », pour employer la formulation d'Ingold (2007:15), et passent d'un état à un autre, changeant de forme, d'apparence, de fonction, de signification et de valeur. L'ontologie et les limites du déchet semblent être par conséquent des effets de performance contextualisés, multiples et instables (Mol 2002) par le biais des différents types d'examens et d'interventions effectués. La question centrale est celle de (l'éventuelle) transition: un ordinateur jetéà la poubelle n'a pas de valeur « pour ce qu'il est, mais pour ce qu'il pourrait devenir» (Gregson et al. 2010 : 853). Les ramasseurs de déchets ne travaillent pas avec certitude et dans la «réalité », mais dans le «virtuel» absolu et dans les possibles « devenir», en faisant des objets cassés des défaillances ou encore des restrictions légales de fructueuses opportunités. Cette (re)création, toutefois, n'est pas chose aisée, car elle dépend des compétences techniques, des connaissances et des réseaux personnels, et comporte de nombreux risques, notamment à s'affranchir des règlements municipaux ou à traverser les zones frontalières.

Le travail quotidien et les pratiques épistémiques réalisés par les ramasseurs de déchets en récupérant des déchets électroniques et en les (re)créant montre comment l'innovation (Akrich et al. 2002, Suchman \& Bishop 2000) ne consiste pas nécessairement à trouver des réponses (techniques) à certains besoins ou à des problèmes définis en amont, mais au contraire à problématiser, à mettre en question et à proposer d'autres économies et ordres mondiaux matériels possibles. En ce sens, ce que nous observons à travers ces pratiques de recréation est que 
l'innovation et la créativité sociotechniques ne résultent pas seulement de la conception ou des pratiques de fabrication -largement considérées comme étant des lieux privilégiés pour la création et la production-, mais se situent également dans des actions plus modestes, «reproductives » ou même marginales et illégales telles que le ramassage de déchets et la récupération, la réparation, voire le démontage de vieux appareils électriques. Le type d'innovation né des pratiques des ramasseurs de déchets provient de l'opposition aux effets maintes fois niés et négligés (Jackson 2014) de notre consommation et de l'opposition à ce phénomène et à notre modèle de production non durable, fondé sur l'obsolescence programmée et perceptible des appareils technologiques (Huisman et al. 2008, Maycroft 2009).

Les pratiques observées ont également démontré une innovation socio-économique via la recréation et la prolifération de nouveaux circuits économiques et régimes de valeur informels - fondés sur le ramassage, l'entretien et la réparation - dans un certain nombre d'endroits en marge, par le biais d'objets mis à la décharge et par des «travailleurs» informels et invisibilisés. De cette façon, certains déchets qui étaient sortis du circuit légal ont été réintroduits et transformés en nouvelles ressources. Dans le même temps, de nombreux migrants sans permis de travail, de séjour, ou dépourvus de droits civiques ont formé un groupe prodigieux de travailleurs informels, réalisant un traitement et un recyclage des déchets minutieux et de qualité, que le conseil municipal, comme l'a souligné un ramasseur de déchets, ne pourrait jamais payer. Ces recherches nous ont permis de mesurer à quel point le développement durable et l'amélioration de nos infrastructures communes, telles que les systèmes de gestion et de traitement des déchets, ne dépendent pas uniquement des initiatives du gouvernement et des institutions publiques, comme on a généralement tendance à le croire, mais aussi des activités en marge et subsidiaires.

Pour se faire une idée réelle et dynamique de notre régime spécifique en matière de déchets (Gille 2010), il est nécessaire d'élargir notre champ de vision aux marges, aux pratiques invisibles mais potentiellement innovantes et aux circuits informels souterrains qui contredisent et redéfinissent nos systèmes de rejet des déchets (électroniques) (ibid.) et les redéfinissent. Cela constitue un défi pour l'étude des appareils électroniques et des déchets mis au rebut, et des recherches émergentes mais prolifiques sur les infrastructures. L'histoire de Keita, Marcel et des autres évoque les «outsiders» et l'illégalité, mais se confronte au circuit officiel du traitement et de la gestion des déchets sur de nombreux points: en le combattant, par le fait d'oser ramasser dans les poubelles et dans le voisinage, en l'entretenant et en composant avec lui dans une relation symbiotique, en récupérant et en vendant le matériel ramassé et trié aux ferrailleurs pour être recyclé; ou encore en l'améliorant et l'étendant, en gagnant sa vie tout au long du processus et en revitalisant des circuits économiques internationaux par la vente et les réparations sur des marchés d'occasion africains. Le légal et l'illégal, le formel et l'informel s'entremêlent, chacun s'infiltrant dans l'autre. La légitimité et l'illégitimité sont négociables et ne sont pas nécessairement réparties de façon rigide respectivement entre les acteurs légaux et illégaux. Il est clair que pour comprendre en profondeur un régime spécifique en matière de déchets - une manière contextualisée de répartir les droits, les règles et de produire, de 
diffuser et de transformer les déchets-, il est insuffisant de se pencher sur ces seuls instruments, infrastructures et pratiques institutionnels et formels: il s'agit aussi de prêter attention à ces acteurs et pratiques informels, inconnus ou volontairement cachés, invisibilisés ou « oubliés », qui parcourent discrètement nos rues ${ }^{10}$.

\section{Notes}

1. Directive 2002/96/CE du Parlement européen et du Conseil du 27 janvier 2003 relative aux déchets d'équipements électroniques et électriques: eur-lex.europa.eu/legal-content/FR/TXT/ HTML/?uri=CELEX:32002L0096\&from=ES.

2. Le 2 juin 2011, un rapport publié dans un journal national annonçait que le ministère public enquêtait sur une fraude massive dans le domaine du recyclage des DEEE: en Espagne, il y a vingt usines de traitement des déchets, mais toutes sont pratiquement vides. Où sont les restes? Voir elpais.com/diario/2011/06/02/ sociedad/1306965607_850215.html.

3. Les deux autres expériences sont intitulées Obsoletos (obsoletos.org/), un projet de recherche sur des hackers et des fabricants, et Cyclica-Labdoo (blogs. latabacalera.net/cyclicka/ et www.labdoo.org/), un atelier autogéré de réparation d'ordinateurs obsolètes collaborant avec un réseau social de donations, tous deux situés à Madrid.

4. Ce travail ethnographique de terrain à Barcelone a eu lieu entre le 15 novembre et le 15 décembre 2012. Durant cette période, j'ai eu recours à une observation participative dans l'entrepôt où vivait et travaillait un groupe de ramasseurs de déchets. J'ai également accompagné l'un d'entre eux de rue en rue, durant certaines de ses journées de travail Au cours de ces journées, j'ai effectué des entretiens individuels avec plusieurs ramasseurs de déchets, des ferrailleurs et des «intermédiaires » au sujet de leurs tâches et de leur travail sur les déchets électroniques et électriques. J'ai pris des photos de leurs procédés de travail, de leurs outils et des endroits qu'ils fréquentaient. Ce complexe d'entrepôts a été évacué durant l'été 2013.

5. La plupart des ramasseurs de déchets d'origine subsaharienne s'intéressent essentiellement au métal, car il rapporte plus, et aux objets suscep- tibles d'être revendus sur des marchés d'occasion clandestins, tels que les habits et chaussures ou toutes sortes d'appareils, outils et objets décoratifs. D'autres ramasseurs de déchets, originaires pour la plupart d'Europe de l'Est, ramassent également le carton. Aujourd'hui, quatre ans après mon travail de terrain, on peut percevoir un changement, avec davantage de personnes ramassant toutes sortes de matériaux: métal, papier et carton. En fait, ce phénomène avait déjà été annoncé par Marcel, notre informateur, lorsqu'il s'était plaint du nombre croissant de ramasseurs de déchets, y compris des gens du coin, et la perte de revenus qui en découlait. Il envisageait alors de commencer à ramasser également le papier et le carton.

6. Comme c'est le cas à Barcelone.

7. Plus la quantité de métal est grande, plus le prix à payer est cher.

8. Uniquement lorsque l'alimentation électrique -branchée sur le réseau public et intermittent-le permet. [NdlT: «Cannibaliser» est un terme technique désignant le fait de récupérer les pièces d'un appareil pour en réparer un autre].

9. Trois ans après mon travail de terrain, en mars 2015, et profitant de l'offre par le ministère de vols gratuits dans le cadre d'un rapatriement volontaire, Marcel décida de retourner au Cameroun, car il ne trouvait pas de travail (il ne possédait pas de permis de travail) et était fatigué de sa vie de ramasseur de déchets.

10. Ces travaux ont été rendus possibles grâce au financement de mon séjour à l'Université de Lancaster, en 2011, par le Spanish Subprogram for Postdoctoral Mobility in Foreign Centers. Je tiens à remercier tout particulièrement les personnes de Obsoletos, Cyclicka-Labdoo et le groupe de ramasseurs de déchets avec lesquels j'ai collaboré, notamment Marcel, Keita et Tilan. 


\section{I'auteure}

Blanca Callén est enseignante-chercheuse au sein du groupe GREDITS au Bau Design College à Barcelone. Elle enseigne également à la faculté de psychologie de l'université de Vic. Actuellement, elle participe au projet européen intitulé « Manufactories of caring space-time » de la Fondation Antoni Tàpies. Elle est titulaire d'un doctorat en psychologie sociale de l'université autonome de Barcelone (UAB). Cet article fait partie d'un projet postdoctoral intitulé « Scrapping politics : innovation and citizen expertise on reducing, reusing and recycling e-waste » (Politiques de mise à la ferraille: innovation et expertise citoyenne en matière de réduction, de réemploi et de recyclage des déchets électroniques), lancé par le Center for Science Studies à l'université de Lancaster (Royaume-Uni). Elle a cofondé le collectif Restarters - BCN et est membre du MEND*RS International Network.

\section{Iconographie}

Image d'ouverture. Démontage d'un ordinateur cassé.

Crédits photographiques pour l'ensemble des images. (C) Blanca Callén.

\section{Références}

Akrich, M., Callon, M \& B. Latour 2002 «The Key to Success in Innovation. I: The Art of Interessement », International Journal of Innovation Management 6 (2): 187-206.

BOPB 2001 Anunci d'Aprovació Definitiva. Modificació de l'Ordenança sobre l'ús de les vies i els espais públics de Barcelona, Butlletí Oficial de la Província de Barcelona 194: Annex I. 61.

Dant, T. 2010 «The Work of Repair: Gesture, Emotion and Sensual Knowledge», Sociological Research Online: www.socresonline.org.uk/15/3/7.html.

Dant, T. \& D. Bowles 2003 « Dealing with Dirt: Servicing and Repairing Cars», Sociological Research Online: www.socresonline.org.uk/8/2/dant.html.

Denis, J. \& D. Pontille 2014 « Maintenance Work and the Performativity of Urban Inscriptions: The Case of Paris Subway Signs », Environment and Planning D: Society and Space 32 (3): 404-416.

Gille, Z. 2010 «Actor Networks, Modes of Production, and Waste Regimes: Reassembling the Macro-Social», Environment and Planning A 42 (5): 1049-1064.

Gregson, N., Crang, M., Ahamed, F., Akhter, N. \& R. Ferdous 2010 «Following Things of Rubbish Value: End-Of-Life Ships, "chock-chocky" Furniture and the Bangladeshi Middle Class Consumer», Geoforum 41 (6): 846-854.

\section{Pour citer cet article}

Callén, B. 2016 «Donner une seconde vie aux déchets électroniques. Économies informelles et innovation sociotechnique des marches», TechniquesE Culture 65-66 «Réparer le monde. Excès, reste et innovation», p. 206-219.
Huisman, J. et al. 2008 Review of Directive 2002/96 on Waste Electrical and Electronic Equipment (WEEE). Bonn: United Nations University.

Ingold, T. 2013 Making: Anthropology, Archaeology, Art and Architecture. New York: Routledge.

— 2007 «Materials Against Materiality», Archaeological Dialogues 14 (1): 1-16.

Jackson, S.J. 2014 «Rethinking Repair» in T. Gillespie, P. Boczkowski \& K. Foot dir. Mia Technologies: Essays on Communication, Materiality and Society. Cambridge: MIT Press: 221-239.

Khurrum, S., Bhutta, M., Omar, A. \& X. Yang 2011 «Electronic Waste: A Growing Concern in Today's Environment», Economics Research International: 1-8.

Lepawsky,J. \& C. Mather 2011 «From Beginnings and Endings to Boundaries and Edges: Rethinking Circulation and Exchange through Electronic Waste», Area 43 (3): 242-249.

Maycroft, N. 2009 Consumption, Planned Obsolescence and Waste. [En ligne] eprints.lincoln.ac.uk/2062/1/Obsolescence.pdf.

Mol, A. 2002 The Body Multiple: Ontology in Medical Practice. Durham: Duke University Press.

Queiruga, D., González Benito, J. \& G. Lannelongue 2012 «Evolution of the Electronic Waste Management System in Spain », Journal of Cleaner Production 24: 56-65.

Suchman, L. \& L. Bishop 2000 «Problematizing "Innovation" as a Critical Project », Technology Analysis and Strategic Management 12 (3): 327-333. 